\title{
The effects of tidal volume size and driving pressure levels on pulmonary complement activation: an observational study in critically ill patients
}

\author{
Friso M. de Beer ${ }^{1,2,3^{*}} \mathbb{0}$, Luuk Wieske ${ }^{1,2,4}$, Gerard van Mierlo ${ }^{6}$, Diana Wouters ${ }^{6}$, Sacha Zeerleder ${ }^{5,6}$, \\ Lieuwe D. Bos ${ }^{1,2}$, Nicole P. Juffermans ${ }^{1,2}$, Marcus J. Schultz ${ }^{1,2,7,8}$, Tom van der Poll ${ }^{9,10}$, Wim K. Lagrand ${ }^{1,2}$, \\ Janneke Horn ${ }^{1,2}$ and for the BASIC-study group
}

From 4th International Symposium on Acute Pulmonary Injury and Translational Research - INSPIRES 2019 Dresden, Germany. 25-26 November 2019

*Correspondence:

frisodebeer@hotmail.com

${ }^{1}$ Department of Intensive

Care Medicine, Amsterdam

UMC, University

of Amsterdam, Amsterdam,

The Netherlands

Full list of author information

is available at the end of the

article

\begin{abstract}
Background: Mechanical ventilation can induce or even worsen lung injury, at least in part via overdistension caused by too large volumes or too high pressures. The complement system has been suggested to play a causative role in ventilator-induced lung injury.
\end{abstract}

Aims and methods: This was a single-center prospective study investigating associations between pulmonary levels of complement activation products and two ventilator settings, tidal volume $\left(V_{T}\right)$ and driving pressure $(\triangle P)$, in critically ill patients under invasive ventilation. A miniature bronchoalveolar lavage (BAL) was performed for determination of pulmonary levels of $\mathrm{C} 5 \mathrm{a}, \mathrm{C} 3 \mathrm{~b} / \mathrm{c}$, and $\mathrm{C} 4 \mathrm{~b} / \mathrm{c}$. The primary endpoint was the correlation between BAL fluid (BALF) levels of $C 5 a$ and $V_{T}$ and $\triangle P$. Levels of complement activation products were also compared between patients with and without ARDS or with and without pneumonia.

Results: Seventy-two patients were included. Median time from start of invasive ventilation till BAL was 27 [19 to 34] hours. Median $V_{\text {T }}$ and $\triangle P$ before BAL were 6.7 [IQR 6.1 to 7.6$] \mathrm{ml} / \mathrm{kg}$ predicted bodyweight (PBW) and 15 [IQR 11 to 18] $\mathrm{cm} \mathrm{H}_{2} \mathrm{O}$, respectively. BALF levels of $\mathrm{C} 5 \mathrm{a}, \mathrm{C} 3 \mathrm{~b} / \mathrm{c}$ and $\mathrm{C} 4 \mathrm{~b} / \mathrm{c}$ were neither different between patients with or without ARDS, nor between patients with or without pneumonia. BALF levels of C5a, and also $\mathrm{C} 3 \mathrm{~b} / \mathrm{c}$ and $\mathrm{C} 4 \mathrm{~b} / \mathrm{c}$, did not correlate with $V_{\mathrm{T}}$ and $\triangle P$. Median BALF levels of $\mathrm{C} 5 \mathrm{a}, \mathrm{C} 3 \mathrm{~b} / \mathrm{c}$, and $\mathrm{C} 4 \mathrm{~b} / \mathrm{c}$, and the effects of $V_{T}$ and $\triangle P$ on those levels, were not different between patients with or without ARDS, and in patients with or without pneumonia.

Conclusion: In this cohort of critically ill patients under invasive ventilation, pulmonary levels of complement activation products were independent of the size of $V_{T}$ and the level of $\triangle P$. The associations were not different for patients with ARDS or with author(s) and the source, provide a link to the Creative Commons licence, and indicate if changes were made. The images or other third party material in this article are included in the article's Creative Commons licence, unless indicated otherwise in a credit line to the material. If material is not included in the article's Creative Commons licence and your intended use is not permitted by statutory regulation or exceeds the permitted use, you will need to obtain permission directly from the copyright holder. To view a copy of this licence, visit http:// creativecommons.org/licenses/by/4.0/. 
pneumonia. Pulmonary complement activation does not seem to play a major role in VILI, and not even in lung injury per se, in critically ill patients under invasive ventilation.

Keywords: Intensive care, Critical care, Mechanical ventilation, Tidal volume, Driving pressure, Bronchoalveolar lavage, Complement, Complement activation, Complement component 5, C5a

\section{Background}

Invasive ventilation has a strong potential to cause so-called ventilator-induced lung injury (VILI) [1], at least in part via overdistension of lung units due to the use of too large volumes or too high pressures [2]. Ventilation with a low tidal volume $\left(V_{\mathrm{T}}\right)$ of $6 \mathrm{ml} /$ $\mathrm{kg}$ predicted body weight (PBW) clearly improves outcomes in patients with acute respiratory distress syndrome (ARDS) [3], and maybe also in patients without ARDS [4, 5]. Ventilation with a high driving pressure $(\Delta P)$, the difference between plateau pressure and positive end-expiratory pressure (PEEP), has been associated with worse outcomes in patients with ARDS [6, 7], and a cut-off value of $15 \mathrm{~cm} \mathrm{H}_{2} \mathrm{O}$ for $\Delta P$ has been proposed and is currently widely used at the bedside as a safety limit.

The pathophysiological mechanisms of VILI remain only partly understood. Complement activation has been suggested as one pathogenetic factor in VILI [8]. However, evidence for a key role of complement activation persists to be poor and originates mainly from preclinical studies. In rats with Streptococcus pneumoniae pneumonia, ventilation with a high $V_{\mathrm{T}}$ increased pulmonary levels of the complement activation product C4b/c [9]. In healthy mice, ventilation with a high $V_{\mathrm{T}}$ resulted in increased complement C3 deposition in the lung and reduced cell aggregation [10]. In healthy rats, ventilation with a high $V_{\mathrm{T}}$ increased pulmonary vascular permeability, a finding that was linked to increased plasma levels of soluble terminal complement complex [11]. Also, in a study with healthy rats, ventilation with high airway pressures resulted in increased C3a levels in plasma [12]. Studies in the human setting are even more scarce, and findings in these studies are conflicting with those in preclinical studies. For instance, no association was found between complement depositions in lung tissue and $\Delta \mathrm{P}$ in critically ill patients who died under invasive ventilation [13].

We initiated the current study to gain a better understanding of the effects of $V_{\mathrm{T}}$ and $\Delta P$ on complement activation in the pulmonary compartment of critically ill patients under invasive ventilation. The hypothesis was that pulmonary complement activation is associated with $V_{\mathrm{T}}$ and $\Delta P$, and also that pulmonary levels of complement activation products are higher in patients with ARDS or with pneumonia.

\section{Methods}

\section{Study design and ethical concerns}

This was a sub-study of the prospective observational 'Biomarker Analysis in Septic Intensive Care patients' (BASIC) study. BASIC was a single-center investigation performed in the intensive care unit (ICU) of the Amsterdam University Medical Centers, location 'AMC', Amsterdam, The Netherlands. The study protocol of BASIC was approved by the Institutional Review Board (METC 2010_335\#B201112). The study was registered at the Dutch Central Commission for Human bound Research (CCMO) 
(study identifier NL34294.018.10). Patients were included from April 2011 to November 2013. Written informed consent was obtained from all patients or their next of kin, before study entry.

\section{Patients}

Patients were eligible for participation in BASIC if: (a) aged 18 years or older; (b) expected ICU stay of at least $24 \mathrm{~h}$; and (c) having at least two criteria for systemic inflammatory response syndrome (SIRS) [14], with or without infectious causality. Readmitted patients, patients who were referred to the participating ICU from an ICU in another hospital, patients treated with antibiotics for $>48 \mathrm{~h}$, patients included in other studies testing interventions that could possibly affect inflammatory processes, and patients of whom no written informed consent was obtained were excluded. For the current analysis, we included patients who were under invasive ventilation and were subjectable to a bronchoalveolar lavage (BAL) (see below). Patients were also excluded if there was no blood sample taken at the moment of the BAL, or if BAL fluid (BALF) was of insufficient quality (see below for definition).

\section{Clinical data and ventilator variables and parameters}

A dedicated team of trained researchers collected baseline characteristics and outcomes, and scored presence of ARDS according to the Berlin definition for ARDS [15]. Presence of pneumonia was assessed using the Centers for Disease Control and Prevention and International Sepsis Forum consensus definitions [16]. Patients with pneumonia on admission and patients having pneumonia within $48 \mathrm{~h}$ of start of ventilation, were considered pneumonia patients.

Granular ventilation data were collected from the electronic patient data monitoring system that recorded and stored (a) ventilation mode, (b) expired $V_{\mathrm{T}}$, (c) PEEP and (d) maximum, peak and plateau airway pressures, and (e) fraction of inspired oxygen $\left(\mathrm{FiO}_{2}\right)$ every $5 \mathrm{~min}$.

\section{BAL}

Within $48 \mathrm{~h}$ after ICU admission a miniature BAL was performed as described before [17]. In short, a 50-cm 14-gauge tracheal suction catheter was inserted via the orotracheal tube and advanced until significant resistance was encountered. Then $20 \mathrm{ml}$ of sterile normal saline was instilled over a period of 4 to $5 \mathrm{~s}$. Immediately hereafter, fluid was aspirated, typically recovering 4 to $8 \mathrm{ml}$. This BALF was processed directly and centrifuged at $1.500 \times g$ for $15 \mathrm{~min}$ at $4{ }^{\circ} \mathrm{C}$; samples were stored at $-80{ }^{\circ} \mathrm{C}$ until assays were performed batchwise.

\section{Assays}

The following complement activation products were measured in BALF to determine complement activation. C5a was measured using a commercial enzyme-linked immunosorbent assays (MicroVue, Quidel, San Diego, CA). C3b/c and C4b/c were measured using home-made enzyme-linked immunosorbent assays (Sanquin, Amsterdam, The Netherlands) as described before [18, 19]. As these assays do not distinguish, 
respectively, $\mathrm{C} 3 \mathrm{~b}$ from $\mathrm{C} 3 \mathrm{bi}$ and $\mathrm{C} 3 \mathrm{c}$, and $\mathrm{C} 4 \mathrm{~b}$ from $\mathrm{C} 4 \mathrm{bi}$ and $\mathrm{C} 4 \mathrm{c}$, we referred to these as $\mathrm{C} 3 \mathrm{~b} / \mathrm{c}$ and $\mathrm{C} 4 \mathrm{~b} / \mathrm{c}$.

Urea levels were determined in BALF and plasma using quantitative colorimetric urea determination (BioAssay Systems, Hayward, CA).

\section{Primary and secondary endpoints}

The coprimary endpoint was the association between complement activation product C5a in BALF and median $V_{\mathrm{T}}$ and median $\triangle P$ from start of invasive ventilation till the BAL.

Other endpoints were the association between pulmonary levels of C5a and median $V_{\mathrm{T}}$ and $\Delta P$ in the 6-h time-frame before BAL, associations between other complement activation products and these two ventilator settings, and local levels of C5a in patients with ARDS versus patients without ARDS, and in patients with pneumonia versus patients without pneumonia.

\section{Power calculation}

Data on which we could base a power calculation were lacking. Therefore, we used samples from all patients who were included in the BASIC study, who were under invasive ventilation and underwent a BAL within $48 \mathrm{~h}$ after its initiation. With 72 analyzable patients, we have a two-sided significance level of 0.05 and a power of $80 \%$ for correlation coefficient $(r)$ as low as 0.325 .

\section{Analysis plan}

Continuous variables were presented as median (25th-75th interquartile range [IQR]) or mean with standard deviation (SD), where appropriate. Categorical variables are shown in proportions (\%). Continuous variables were analyzed using a Mann-Whitney $U$-test or Student's $t$-test according to data distribution, proportions were compared using a Fisher exact test. From the available 5-min ventilation data, first the median Vt, $\triangle P$ and PEEP level was calculated for each patient. Then these medians were used to calculate the median and IQR of the whole group. $V_{\mathrm{T}}$ was expressed in $\mathrm{ml} / \mathrm{kg}$ predicted bodyweight (PBW) [20]. $\Delta P$ was calculated by subtracting PEEP from the maximum airway pressure, as all patients were under pressure-controlled modes of ventilation and no plateau airway pressures were available $[21,22]$.

To correct BALF levels of $\mathrm{C} 5 \mathrm{a}, \mathrm{C} 3 \mathrm{~b} / \mathrm{c}$, and $\mathrm{C} 4 \mathrm{~b} / \mathrm{c}$ for differences caused by dilution of the samples, the ratio between urea in BALF and urea in plasma was used to correct complement concentrations in BALF, as described before [23]. Patients with 'low quality' BALF, defined as samples with urea levels below the detection limit, were excluded from the analyses. Levels were presented for the whole group, and for patients with ARDS or pneumonia. Levels were individually plotted on a log-scale together with Tukey boxplots.

The association between levels of pulmonary complement activation and $V_{\mathrm{T}}$ and $\Delta \mathrm{P}$ was analyzed in two ways. First, the association with $V_{\mathrm{T}}$ and $\Delta P$ from start of invasive ventilation till BAL was investigated. Also, the association with ventilation in the $6 \mathrm{~h}$ before BAL was investigated. We used scatterplots and $r$ using Pearson's/Spearman association method according to data distribution. The complete analysis was repeated to 
compare the effects of ventilator settings on complement activation products in patients with ARDS versus those without ARDS, and in patients with pneumonia and patients without pneumonia.

Statistical analyses were performed using GraphPad Prism version 8.0.2 (GraphPad software Inc, La Jolla, CA, USA). A $P$-value of $<0.05$ was considered statistically significant.

\section{Results}

\section{Patients}

Flowchart of patients is shown in Fig. 1. In total, 355 patients were included in the BASIC study. After excluding patients who were not under invasive ventilation, patients who did not have a matched BALF to plasma sample, and patients in whom BALF was considered of poor quality, 72 patients remained for the current analysis. Of these patients, 21 patients (29\%) were classified as having ARDS, and 29 patients (40\%) had pneumonia. Patient characteristics are presented in Table 1. ICU mortality rate was $25 \%$.

Median time from start of invasive ventilation to BAL was 27 [IQR 19 to 34] hours. Median $V_{\mathrm{T}}$ from start of ventilation to BAL was 6.7 [IQR 6.1 to 7.6 ] $\mathrm{ml} / \mathrm{kg}$ PBW, comparable to the median $V_{\mathrm{T}}$ of 6.8 [IQR 6.1 to 8.0 ] $\mathrm{ml} / \mathrm{kg} \mathrm{PBW}$ in the last $6 \mathrm{~h}$ before the

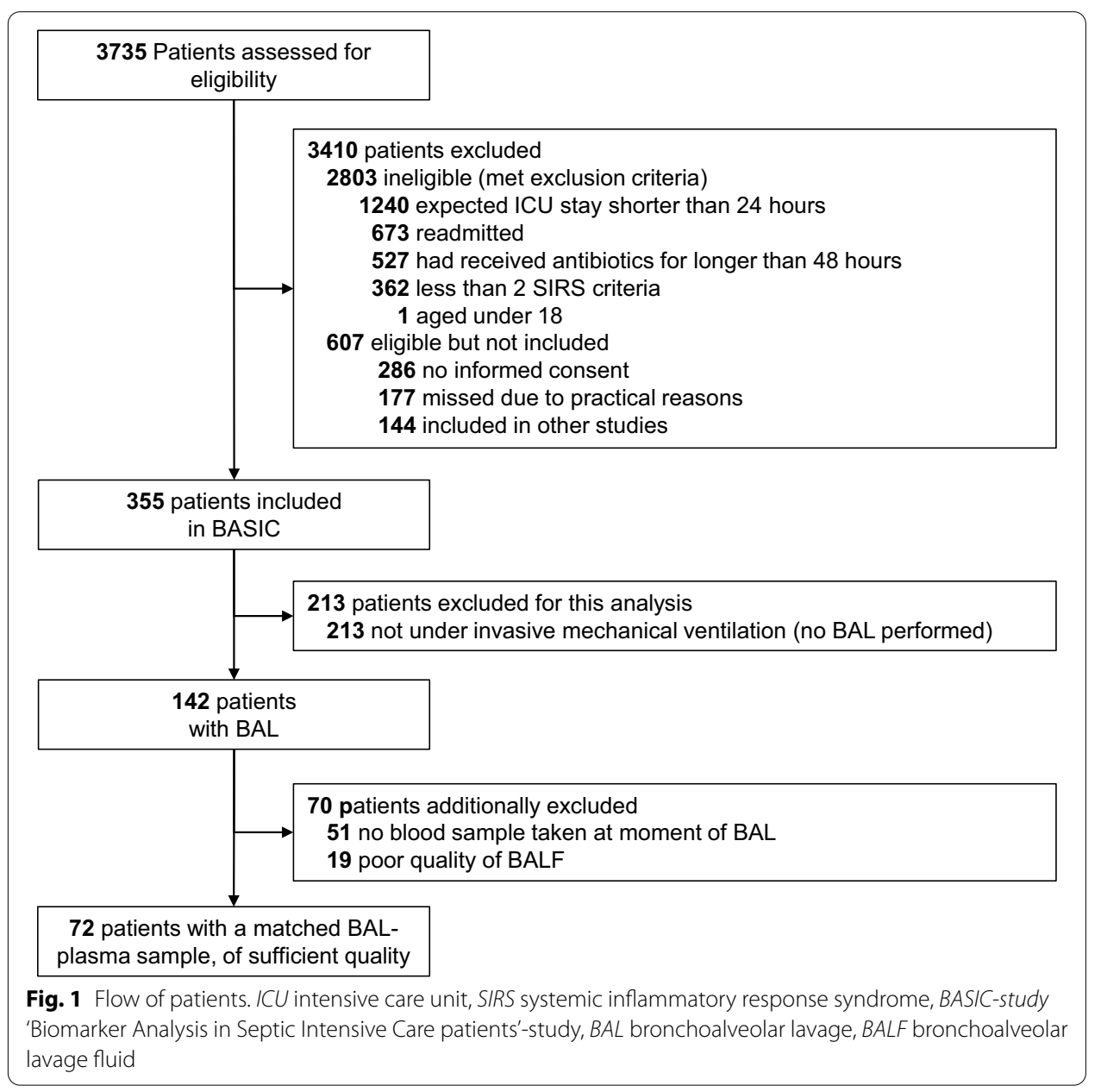


Table 1 Patient characteristics

\begin{tabular}{|c|c|c|}
\hline & $\begin{array}{l}\text { Patients } \\
(N=72)\end{array}$ & \\
\hline Age (years), median [IQR] & 62.0 & {$[49.3-72.8]$} \\
\hline Female gender, $n(\%)$ & 32 & $(44.4)$ \\
\hline Weight $(\mathrm{kg})$, median [IQR] & 80.0 & {$[65.0-90.0]$} \\
\hline Length $(\mathrm{cm})$, median $[\mathrm{IQR}]$ & 175.0 & {$[165.3-180.0]$} \\
\hline $\mathrm{BMI}\left(\mathrm{kg} / \mathrm{m}^{2}\right)$, median $[\mathrm{IQR}]$ & 25.6 & {$[22.7-29.7]$} \\
\hline \multicolumn{3}{|l|}{ ICU admission type } \\
\hline Medical, n (\%) & 58 & $(80.6)$ \\
\hline Surgical emergency, n (\%) & 10 & $(13.9)$ \\
\hline Surgical elective, n (\%) & 4 & $(5.6)$ \\
\hline \multicolumn{3}{|l|}{ Severity of illness } \\
\hline Max SOFA score, median [IQR] & 10.0 & {$[8.0-13.0]$} \\
\hline APACHE IV score, median [IQR] & 84.5 & {$[67.0-107.0]$} \\
\hline SAPS, median [IQR] & 54.5 & {$[47.0-70.3]$} \\
\hline \multicolumn{3}{|l|}{ Lung injury at start of ventilation } \\
\hline ARDS, n (\%) & 21 & $(29.2)$ \\
\hline Pneumonia, n (\%) & 29 & $(40.3)$ \\
\hline Lowest $\mathrm{PaO}_{2}: \mathrm{FiO}_{2}$, median $[\mathrm{IQR}]$ & 172 & {$[145-220]$} \\
\hline \multicolumn{3}{|l|}{ Comorbidities } \\
\hline COPD, n (\%) & 4 & $(5.6)$ \\
\hline Sepsis, n (\%) & 43 & $(58.3)$ \\
\hline \multicolumn{3}{|l|}{ ICU outcomes } \\
\hline ICU length of stay (days), median [IQR] & 6.5 & {$[4.0-10.0]$} \\
\hline Total MV days, median [IQR] & 4.0 & {$[2.8-8.3]$} \\
\hline ICU mortality, n (\%) & 18 & $(25.0)$ \\
\hline
\end{tabular}

$B M I$ body mass index, SOFA Sequential Organ Failure Assessment, APACHE Acute Physiology and Chronic Health Score, SAPS Simplified Acute Physiology Score, ARDS acute respiratory distress syndrome, COPD chronic obstructive pulmonary disease

BAL. Median $\triangle \mathrm{P}$ from start of ventilation to $\mathrm{BAL}$ was 15 [IQR 11 to 18 ] $\mathrm{cmH}_{2} \mathrm{O}$, slightly higher than the median $\Delta \mathrm{P}$ of 13 [IQR 9 to 18] $\mathrm{cmH}_{2} \mathrm{O}$ in the last $6 \mathrm{~h}$ before BAL. Median PEEP from start of ventilation to BAL was 7.8 [IQR 5.0 to 10.0] $\mathrm{cmH}_{2} \mathrm{O}$, higher than the median PEEP of 6.0 [IQR 5.0 to 10.0] $\mathrm{cmH}_{2} \mathrm{O}$ in the last $6 \mathrm{~h}$ before BAL.

\section{BALF levels of complement activation products}

Median levels of complement activation products in BALF are shown in Fig. 2. Median C5a level was 103 [IQR 49-307] ng/ml, median C3b/c and C4b/c levels were 739 [IQR 471-1939] and 79 [IQR 38-179] nmol/L, respectively. Complement activation products in BALF were similar in patients with ARDS or without ARDS. The same was found for patients with or without pneumonia.

\section{Correlations between pulmonary complement activation and $V_{T}$ and $\Delta P$}

The correlation between BALF levels of complement activation product C5a and $V_{\mathrm{T}}$ and $\triangle P$ was poor, as illustrated in Fig. 3. Correlations were also poor between BALF levels of the other two complement components and median $V_{\mathrm{T}}$ and median $\Delta P$.

Restricting the analysis to ventilation data collected within the last $6 \mathrm{~h}$ before the lavage, did not change the results (see Additional file 1: Figure S1). Comparing 

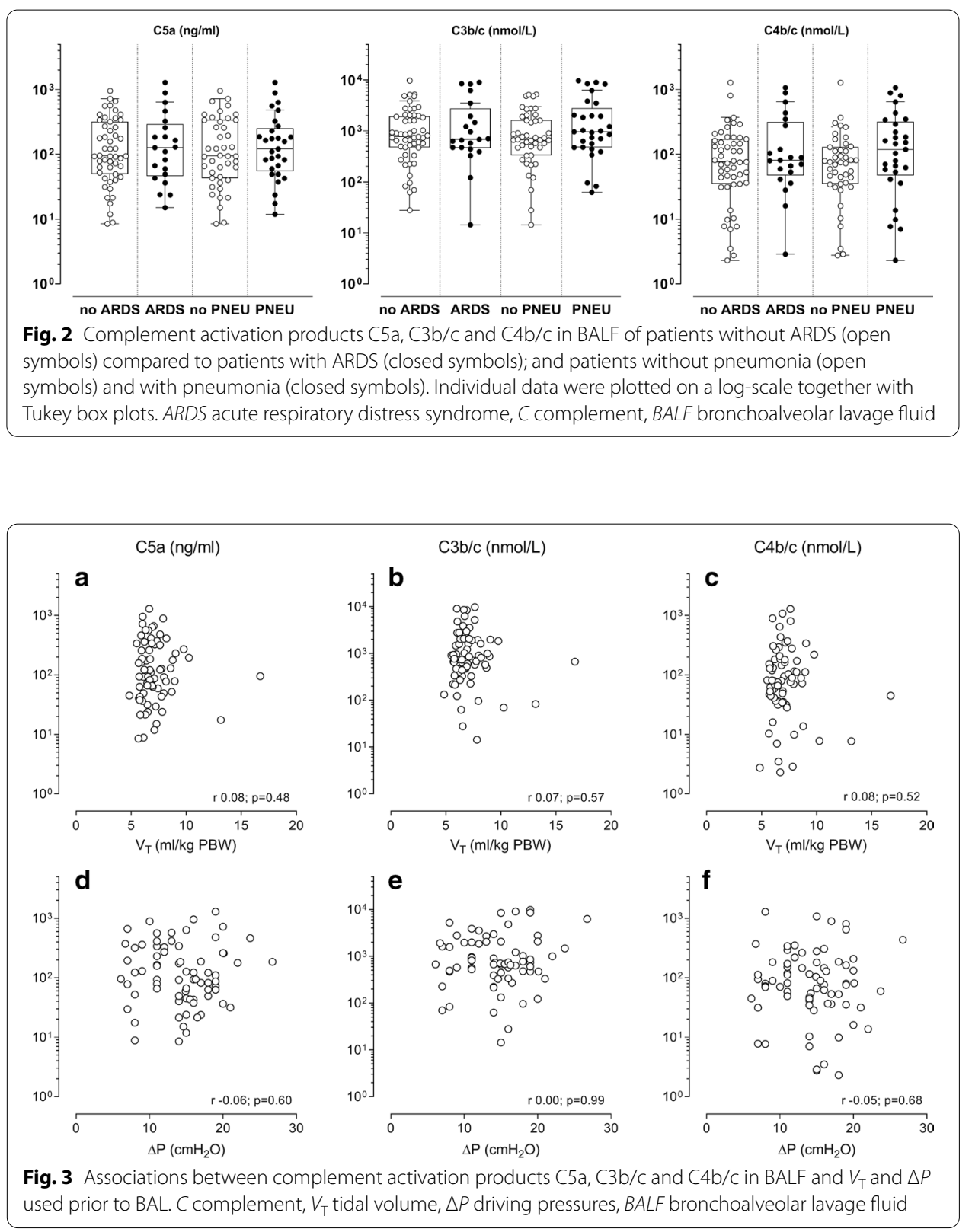

associations between $V_{\mathrm{T}}$ and $\Delta P$ in patients with ARDS versus patients without ARDS, and in patients with or without pneumonia resulted in comparable findings (see Additional file 2: Figure S2 and Additional file 3: Figure S3).

\section{Discussion}

The results of this study in a cohort in critically ill patients under invasive ventilation for various reasons can be summarized as follows: (a) no association was found between pulmonary levels of complement activation products and two main ventilator settings, $V_{\mathrm{T}}$ and $\Delta P$, and (b) this was neither different for patients with ARDS and patients without ARDS, nor for patients with pneumonia and patients without 
pneumonia; (c) one other salient finding was that pulmonary levels of complement activation products were similar between patients with and patients without ARDS or pneumonia.

This study knows several strengths. Bias was minimized by its prospective character, and a preplanned analysis plan was strictly followed. The clear inclusion and exclusion criteria led to a recognizable population of critically ill patients with high severity of illness scores. The number of included patients was large, exceeding the numbers of several preceding studies. Patients underwent a BAL relatively soon after start of invasive ventilation. Last but not least, the electronic patient data monitoring system allowed us to use granular ventilation data, providing an accurate reflection of ventilator settings before BAL in all patients.

Since complement factors are unstable, we payed extensive attention to proper handling of the BAL samples. Samples were immediately processed and stored. All analyses were performed batchwise. Therefore, an effect of complement instability on our results is very unlikely. With a miniature BAL, differences in dilution can result in erroneously low levels of biomarkers of interest, in this case complement activation products. Differences in urea concentration in BAL and plasma were used to correct for this inaccuracy [23]. We improved the quality of our data collection by removing samples from the final analysis when BALF urea levels suggested too much dilution.

Though no data exist on levels of complement activation products in BALF in humans with VILI, several studies have been performed in patients with ARDS [24-26]. One of these studies used C5a as the marker of complement activation, alike we used in the current investigation [24]. In that study, median levels of C5a in BALF were $400 \mathrm{ng} /$ $\mathrm{ml}$, fourfold higher than the median level found in our study. It should be noted, though, that there were important differences in sampling (a miniature BAL in the current study vs a formal BAL in the previous one) as well as sample handling (no further processing in the current study versus concentrating supernatant using pressure filtration. Last but not least, the two investigations used different assays for C5a measurements.

The results of the current investigation are in contrast with findings in previous animal studies [9-12]. In rodent models of VILI, ventilation with a high $V_{\mathrm{T}}$ [9-11] or a high ventilation pressure [12] resulted in clear complement activation, seen the increased levels of complement activation products. It must be mentioned, that in those studies VILI was induced by ventilation with a high $V_{\mathrm{T}}(12 \mathrm{ml} / \mathrm{kg})$ [9] to an extreme high $V_{\mathrm{T}}(35 \mathrm{ml} /$ $\mathrm{kg}$ [10] or $40 \mathrm{ml} / \mathrm{kg}$ [11]), or with ventilation with a high peak pressure, and thus probably a high $\Delta P$ [12]. Those settings no longer reflect current clinical practice. Indeed, in the current study, $V_{\mathrm{T}}$ and $\Delta P$ were all within widely recommended ranges [27, 28].

One other important difference between preceding animal studies and the current human investigation is that in the animal studies identical hits could be used in a wellcontrolled setting in genetically comparable rodents, while the cohort of patients in the current study was heterogeneous and had a variety of pulmonary hits. But even with use of a relatively large cohort of patients, to correct for these variances, no correlation was found between pulmonary levels of complement activation products and the two ventilator settings of interest.

The findings of the current study are in line with one previous human study of our group. In a series of critically ill patients who died under invasive ventilation, we found 
no association between complement $\mathrm{C} 3 \mathrm{~d}$ depositions in lung tissue and $\Delta P$ in the final hours before death [13]. No difference in deposition of C3d in ARDS patients when compared to patients without ARDS was present. Two other studies showed increased BALF levels of complement activation products in ARDS patients [24, 25]. It must be mentioned that in these studies complement levels in patients were compared to levels in healthy volunteers and postoperative patients, and not to levels in critically ill patients without ARDS. In one other study, a difference in levels of pulmonary complement activation was found between trauma patients who developed ARDS and those who did not, but these differences were only present very early after start of ventilation, i.e., within hours after its initiation [26]. It is possible that we were 'too late' to find a difference in complement activation in the current study.

Several limitations need to be mentioned. With the use of medians to reflect $V_{\mathrm{T}}$ and $\Delta P$ during invasive ventilation, shorter periods of more injurious ventilator settings could have been missed. As mentioned above, this also means that we cannot exclude a possible effect of (much) higher $V_{\mathrm{T}}$ and $\Delta P$ on pulmonary complement activation. Use of miniature BAL might be inferior to a formal BAL. However, previous studies showed no differences between these two techniques with regard to, e.g., counts of colony forming units [17]. Miniature BAL has the advantage of being less invasive and is therefore frequently used in studies as alternative to a formal BAL [29-31]. Finally, complement activation within the pulmonary compartment in critically ill patients is possibly already high at baseline. This may partially mask the effects on complement activation of any ventilator setting.

\section{Conclusion}

In this cohort of critically ill patients under invasive ventilation for various reasons, no association between levels of pulmonary complement activation and $V_{\mathrm{T}}$ or $\Delta P$ was found. It could be that pulmonary complement activation does not play a major role in VILI, and not even in lung injury per se. If true, treatment with complement inhibitors may not contribute to a better outcome in critically ill patients under invasive ventilation.

\section{Supplementary information}

Supplementary information accompanies this paper at https://doi.org/10.1186/s40635-020-00356-6.

Additional file 1: Figure S1. Association between complement activation products, $C 5 a(A+D), C 3 b / C(B+E)$ and $\mathrm{C} 4 \mathrm{~b} / \mathrm{c}(\mathrm{C}+\mathrm{F})$ in bronchoalveolar lavage fluid and tidal volume $(\mathrm{A}-\mathrm{C})$ and driving pressure (D-F) in the last $6 \mathrm{~h}$ before BAL. Abbreviations: $C$, complement activation product; $V_{T}$, tidal volume; $\triangle P$, driving pressure.

Additional file 2: Figure S2. Association between complement activation products $C 5 a(A+D), C 3 b / C(B+E)$ and $\mathrm{C} 4 \mathrm{~b} / \mathrm{c}(\mathrm{C}+\mathrm{F})$ in bronchoalveolar lavage fluid and tidal volume $(\mathrm{A}-\mathrm{C})$ and driving pressure $(\mathrm{D}-\mathrm{F})$ in patients with (closed symbols) and patients without acute respiratory distress syndrome (open symbols). Abbreviations: $\mathrm{C}$, complement activation product; $V_{T}$, tidal volume; $\Delta P$, driving pressures.

Additional file 3: Figure S3. Association between complement activation products $C 5 a(A+D), C 3 b / C(B+E)$ and $\mathrm{C} 4 \mathrm{~b} / \mathrm{c}(\mathrm{C}+\mathrm{F})$ in bronchoalveolar lavage fluid and tidal volume $(\mathrm{A}-\mathrm{C})$ and driving pressure $(\mathrm{D}-\mathrm{F})$ in patients with (closed symbols) and patients without pneumonia (open symbols). Abbreviations: $C$, complement activation products; $V_{T}$, tidal volume; $\Delta \mathrm{P}$, driving pressure.

Abbreviations

ARDS: Acute respiratory distress syndrome; BAL: Bronchoalveolar lavage; BALF: Bronchoalveolar lavage fluid; ICU: Intensive care unit; BMI: Body mass index; PBW: Predicted bodyweight; APACHE: Acute Physiology and Chronic Health Evaluation; SAPS: Simplified Acute Physiology Scores; $\mathrm{FiO}_{2}$ : Fraction of inspired oxygen; PEEP: Positive end-expiratory pressure; 
SIRS: Systemic inflammatory response; VILI: Ventilator-induced lung injury; $V_{T}$ :Tidal volume; $\triangle P$ : Driving pressure; IQR: Interquartile range; SOFA: Sequential Organ Failure Assessment; COPD: Chronic obstructive pulmonary disease.

\section{Acknowledgements}

All collaborating authors in the BASIC-study group: de Beer FM, Bos LD, Claushuis TA, Glas GJ, Horn J, Hoogendijk AJ, van Hooijdonk RT, Huson MA, de Jong MD, Juffermans NP, Lagrand WK, van der Poll T, Scicluna B, Schouten LR, Schultz MJ, van der Sluijs KF, Straat M, van Vught LA, Wieske L, Wiewel MA, and Witteveen E (all from Amsterdam University Medical Centers, location AMC, Amsterdam, The Netherlands).

\section{About this supplement}

This article has been published as part of Intensive Care Medicine Experimental Volume 8 Supplement 1, 2020: Proceedings from the Fourth International Symposium on Acute Pulmonary Injury and Translation Research (INSPIRES IV). The full contents of the supplement are available at https://icmexperimental.springeropen.com/articles/supplements/volum e-8-supplement-1.

\section{Authors' contributions}

$F B, T P, N J, J H, M S$ and WL conceived the study. FB, LW and LB performed bronchoalveolar lavages and obtained blood samples. DW and SZ provided antibodies and facility for complement measurements. FB and GM performed the complement measurements. FB and LW analyzed and interpreted the data. FB, JH, MS and WL drafted the manuscript. All authors read and approved the final manuscript.

\section{Funding}

This article did not receive sponsorship for publication.

\section{Availability of data and materials}

The datasets generated during and/or analyzed during the current study are available from the corresponding author on reasonable request.

\section{Ethics approval and consent to participate}

The study protocol of BASIC was approved by the Institutional Review Board (METC 2010_335\#B201112). Written informed consent was obtained from all patients or their next of kin, before study entry.

\section{Consent for publication}

Not applicable.

\section{Competing interests}

The authors declare that they have no competing interest.

\section{Author details}

1 Department of Intensive Care Medicine, Amsterdam UMC, University of Amsterdam, Amsterdam, The Netherlands. ${ }^{2}$ Laboratory of Experimental Intensive Care and Anesthesiology (LEICA), Amsterdam UMC, University of Amsterdam, Amsterdam, The Netherlands. ${ }^{3}$ Department of Anesthesiology, Amsterdam UMC, University of Amsterdam, Mail stop H1-118,Meibergdreef 9, 1105 AZ Amsterdam, The Netherlands. ${ }^{4}$ Department of Neurology, Amsterdam UMC, University of Amsterdam, Amsterdam, The Netherlands. ${ }^{5}$ Department of Hematology, Amsterdam UMC, University of Amsterdam, Amsterdam, The Netherlands. ${ }^{6}$ Department of Immunopathology, Sanquin Research and Landsteiner Laboratory, Amsterdam, The Netherlands. ${ }^{7}$ Mahidol-Oxford Tropical Medicine Research Unit (MORU), Mahidol University, Bangkok, Thailand. ${ }^{8}$ Nuffield Department of Medicine, University of Oxford, Oxford, UK. ${ }^{9}$ Department of Internal Medicine, Amsterdam UMC, University of Amsterdam, Amsterdam, The Netherlands. ${ }^{10}$ Center for Experimental and Molecular Medicine (CEMM), Amsterdam UMC, University of Amsterdam, Amsterdam, The Netherlands.

Received: 4 November 2020 Accepted: 6 November 2020

Published: 18 December 2020

\section{References}

1. Slutsky AS, Ranieri VM (2013) Ventilator-induced lung injury. N Engl J Med 369(22):2126-2136

2. Serpa Neto A, Nagtzaam L, Schultz MJ (2014) Ventilation with lower tidal volumes for critically ill patients without the acute respiratory distress syndrome: a systematic translational review and meta-analysis. Curr Opin Crit Care. 20(1):25-32

3. Putensen C et al (2009) Meta-analysis: ventilation strategies and outcomes of the acute respiratory distress syndrome and acute lung injury. Ann Intern Med 151(8):566-576

4. Serpa Neto A et al (2014) Association between tidal volume size, duration of ventilation, and sedation needs in patients without acute respiratory distress syndrome: an individual patient data meta-analysis. Intensive Care Med 40(7):950-957

5. Serpa Neto A et al (2015) Lung-protective ventilation with low tidal volumes and the occurrence of pulmonary complications in patients without acute respiratory distress syndrome: a systematic review and individual patient data analysis. Crit Care Med 43(10):2155-2163

6. Amato MB et al (2015) Driving pressure and survival in the acute respiratory distress syndrome. N Engl J Med 372(8):747-755

7. Aoyama $\mathrm{H}$ et al (2018) Association of driving pressure with mortality among ventilated patients with acute respiratory distress syndrome: a systematic review and meta-analysis. Crit Care Med 46(2):300-306

8. Chen L et al (2018) Molecular mechanisms of ventilator-induced lung injury. Chin Med J (Engl) 131(10):1225-1231 
9. de Beer FM et al (2014) Plasma-derived human C1-esterase inhibitor does not prevent mechanical ventilationinduced pulmonary complement activation in a rat model of Streptococcus pneumoniae pneumonia. Cell Biochem Biophys. https://doi.org/10.1007/s12013-014-9983-7

10. Takahashi $\mathrm{K}$ et al (2011) Complement 3 is involved with ventilator-induced lung injury. Int Immunopharmacol 11(12):2138-2143

11. Liu K et al (2013) SC5b-9-induced pulmonary microvascular endothelial hyperpermeability participates in ventilatorinduced lung injury. Cell Biochem Biophys 67(3):1421-1431

12. Petersen B et al (2016) Complement activation contributes to ventilator-induced lung injury in rats. J Physiol Pharmacol 67(6):911-918

13. de Beer FM et al (2019) Pulmonary complement depositions in autopsy of critically ill patients have no relation with ARDS. Intensive Care Med Exp 7(Suppl 1):35

14. Bone RC et al (1992) Definitions for sepsis and organ failure and guidelines for the use of innovative therapies in sepsis. The ACCP/SCCM Consensus Conference Committee. American College of Chest Physicians/Society of Critical Care Medicine. Chest 101(6):1644-1655

15. Ranieri VM et al (2012) Acute respiratory distress syndrome: the Berlin Definition. JAMA 307(23):2526-2533

16. Klein Klouwenberg PM et al (2013) Interobserver agreement of Centers for Disease Control and Prevention criteria for classifying infections in critically ill patients. Crit Care Med 41(10):2373-2378

17. $A^{\prime}$ Court $\mathrm{CH}$ et al (1993) Microbiological lung surveillance in mechanically ventilated patients, using non-directed bronchial lavage and quantitative culture. Q J Med 86(10):635-648

18. Bruins $P$ et al (1997) Activation of the complement system during and after cardiopulmonary bypass surgery: postsurgery activation involves $\mathrm{C}$-reactive protein and is associated with postoperative arrhythmia. Circulation 96(10):3542-3548

19. Wolbink GJ et al (1993) Application of a monoclonal antibody against a neoepitope on activated C4 in an ELISA for the quantification of complement activation via the classical pathway. J Immunol Methods 163(1):67-76

20. Acute Respiratory Distress Syndrome $N$ et al (2000) Ventilation with lower tidal volumes as compared with traditional tidal volumes for acute lung injury and the acute respiratory distress syndrome. N Engl J Med 342(18):1301-1308

21. Bos LD et al (2016) External validation of the APPS, a new and simple outcome prediction score in patients with the acute respiratory distress syndrome. Ann Intensive Care 6(1):89

22. Chatburn RL et al (2010) Documentation issues for mechanical ventilation in pressure-control modes. Respir Care 55(12):1705-1716

23. Rennard SI et al (1986) Estimation of volume of epithelial lining fluid recovered by lavage using urea as marker of dilution. J Appl Physiol 60(2):532-538

24. Robbins RA et al (1987) Activation of the complement system in the adult respiratory distress syndrome. Am Rev Respir Dis 135(3):651-658

25. Hallgren R et al (1987) Complement activation and increased alveolar-capillary permeability after major surgery and in adult respiratory distress syndrome. Crit Care Med 15(3):189-193

26. Zilow $\mathrm{G}$ et al (1992) Generation of anaphylatoxin C3a in plasma and bronchoalveolar lavage fluid in trauma patients at risk for the adult respiratory distress syndrome. Crit Care Med 20(4):468-473

27. Bellani $\mathrm{G}$ et al (2016) Epidemiology, patterns of care, and mortality for patients with acute respiratory distress syndrome in intensive care units in 50 countries. JAMA 315(8):788-800

28. Serpa Neto S et al (2016) Epidemiological characteristics, practice of ventilation, and clinical outcome in patients at risk of acute respiratory distress syndrome in intensive care units from 16 countries (PRoVENT): an international, multicentre, prospective study. Lancet Respir Med 4(11):882-893

29. Determann RM et al (2009) Plasma CC16 levels are associated with development of ALI/ARDS in patients with ventilator-associated pneumonia: a retrospective observational study. BMC Pulm Med 9:49

30. Loan HT et al (2012) Semi-recumbent body position fails to prevent healthcare-associated pneumonia in Vietnamese patients with severe tetanus. Trans R Soc Trop Med Hyg 106(2):90-97

31. Schultz MJ et al (2004) Local activation of coagulation and inhibition of fibrinolysis in the lung during ventilator associated pneumonia. Thorax 59(2):130-135

\section{Publisher's Note}

Springer Nature remains neutral with regard to jurisdictional claims in published maps and institutional affiliations. 